\title{
FINITE RATE OF INNOVATION BASED MODELING AND COMPRESSION OF ECG SIGNALS
}

\author{
$\begin{array}{lll}\text { G. Baechler } & \text { N. } \text { Freris }^{\star} \quad \text { R.F. Quick } & \text { R. E. Crochiere }\end{array}$ \\ * School of Computer and Communication Sciences, EPFL, 1015 Lausanne, Switzerland \\ † Qualcomm Inc., 5775 Morehouse Drive, San Diego, CA 92121, USA
}

\begin{abstract}
Mobile health is gaining increasing importance for society and the quest for new power efficient devices sampling biosignals is becoming critical. We discuss a new scheme called Variable Pulse Width Finite Rate of Innovation (VPW-FRI) to model and compress ECG signals. This technique generalizes classical FRI estimation to enable the use of a sum of asymmetric Cauchy-based pulses for modeling electrocardiogram (ECG) signals. We experimentally show that VPW-FRI indeed models ECG signals with increased accuracy compared to current standards. In addition, we study the compression efficiency of the method: compared with various widely used compression schemes, we showcase improvements in terms of compression efficiency as well as sampling rate.
\end{abstract}

Index Terms - Variable Pulse Width, Finite Rate of Innovation, ECG modeling, ECG compression

\section{INTRODUCTION}

The classical sampling theory pioneered by Nyquist and Shannon relies on the assumption that the acquired signals are band-limited to a maximum frequency, the Nyquist frequency. Even if this is not the case, a signal can be low-pass filtered to satisfy this assumption.

Finite Rate of Innovation (FRI) theory [1] constitutes, in essence, a generalization of this assumption: signals under consideration must be representable with a finite number of parameters per unit of time (on average). FRI can recover these parameters to a greater degree of accuracy than might be expected from just the bandwidth of the signal. The atoms of the classical FRI framework are Dirac pulses, which are characterized by time of occurrence and amplitude. It has been shown in [1] that, under proper assumptions, these parameters can be accurately recovered by finding the roots of an annihilating filter for the Fourier series of the signal, regardless of the bandwidth of the sampled signal.

The Variable Pulse Width (VPW)-FRI model was first proposed in [2]. In this paper, we formalize the derivation of the model in both time and frequency domains and further demonstrate how to estimate the parameters using an extension of the classical FRI approach. We also apply the new algorithm to ECG modeling and show that VPW-FRI can capture ECG signals with high accuracy using a moderate number of parameters. Moreover, we show that this model compares favorably with current ECG compression and modeling techniques.

\section{BACKGROUND AND RELATED WORK}

We briefly recap an archetypal example of FRI identification and proceed to review the most commonly used ECG compression techniques.

\subsection{FRI}

A typical signal with finite rate of innovation is a $\tau$-periodic stream of $K$ Diracs:

$$
x(t)=\sum_{n \in \mathbb{Z}} \sum_{k=0}^{K-1} b_{k} \delta\left(t-t_{k}-n \tau\right),
$$

where $\left\{t_{k}\right\}_{k=0}^{K-1}$ represent the locations of the pulses and $\left\{b_{k}\right\}_{k=0}^{K-1}$ the amplitudes. We review how to recover the different parameters $\left\{t_{k}, b_{k}\right\}_{k=0}^{K-1}$ by using at least $2 K$ Fourier series coefficients:

$$
X[m]=\frac{1}{\tau} \sum_{k=0}^{K-1} b_{k} e^{-i 2 \pi t_{k} m / \tau}, \quad m \in \mathbb{Z} .
$$

The first step is to find an annihilating filter $A$ such that

$$
(A * X)[m]=0, \forall m \in \mathbb{Z} .
$$

The annihilating filter coefficients can be found by solving the linear system of equations

$$
-X[m]=A[1] X[m-1]+\cdots+A[k] X[m-K]
$$

for $m=0,1, \ldots K$. It has been shown in [1] that any signal $X[m]$ of the form $\sum_{k=0}^{K-1} b_{k} u_{k}^{m}$, with $b_{k} \in \mathbb{R}$ and $u_{k} \in \mathbb{C}$ is annihilated by the filter

$$
A(z)=\sum_{k=0}^{K} A[k] z^{-k}=\prod_{k=0}^{K-1}\left(1-u_{k} z^{-1}\right) .
$$


From (1), we see that the roots $\left\{u_{k}\right\}_{k=0}^{K-1}$ of the Z-transform of the filter annihilating $X[\mathrm{~m}]$ are all located on the unit circle in the complex plane. Knowledge of these roots enables the recovery of the time locations of the Diracs by $t_{k}=-\frac{\tau \angle u_{k}}{2 \pi}$, where $\angle u_{k}$ denotes the phase of $u_{k}$. Note that this is a nonlinear estimation method as it requires the computation of the roots. Once we know the $t_{k}$ 's, we can simply find the amplitudes $\boldsymbol{b}=\left[b_{0}, b_{1}, \ldots, b_{K-1}\right]^{T}$ by solving the following set of linear equations:

$$
\frac{1}{\tau} \boldsymbol{V} \boldsymbol{b}=\boldsymbol{x}
$$

where

$$
\boldsymbol{V}=\left[\begin{array}{cccc}
1 & 1 & \cdots & 1 \\
u_{0} & u_{1} & \cdots & u_{K-1} \\
\vdots & \vdots & \ddots & \vdots \\
u_{0}^{K-1} & u_{1}^{K-1} & \cdots & u_{K-1}^{K-1}
\end{array}\right]
$$

and $\boldsymbol{x}=[X[0], X[1], \ldots, X[K-1]]^{T} ; \boldsymbol{V}$ is a Vandermonde matrix and is thus invertible when all $u_{k}$ 's are distinct. Note that we can pick any set of consecutive Fourier coefficients, but considering $X[m]$ for $m=-K-1, \ldots, K$ results to minimizing the sampling frequency.

\subsection{Prior work on ECG compression and modeling}

There is a wealth of generic signal compression (both lossless and lossy) methods but there are also techniques specifically applicable to ECG signals. An elaborate overview of ECG compression algorithms can be found in [3]. ECG lossy compression methods are usually classified into two main categories: direct methods and transform-based methods. Direct methods are applied in the time domain: for instance, Amplitude Zone Time Epoch Coding (AZTEC) [4] is an algorithm that converts ECG signals into a sequence of piecewise affine functions. Transform-based techniques seek for a sparse representation in a particular basis and compression is achieved by discarding coefficients with magnitude less than a certain threshold. Typical examples include the Fourier transform, the Karuhnen-Loève transform, or the wavelet transform [5]. Compressive sensing (CS) has also been investigated: a practical implementation using Daubechies wavelets is presented in [6].

A first FRI-based modeling of ECG signals is exposed in [7]: heart beats are split into two parts, a) the $Q R S$ complex, which is modeled using a non-uniform spline of order 1 , and b) the residual, which is sampled at low rate (around 10 $\mathrm{Hz}$ ). Our work differs from [7] in two different aspects. First, in VPW-FRI, the entire heart beat is modeled and estimated with FRI techniques, as opposed to simply the QRS complex in [7]. Second, our work relies on asymmetric Cauchy-based pulses, each characterized with four parameters, while in [7], the underlying model is a linear transformation of a sum of Diracs (second derivative of a first order spline). VPW-FRI attains better modeling accuracy as well as higher resilience to noise.

Other pulse shapes can be used to model ECG signals: for example, McSharry et al. [8] proposed Gaussian mixtures to synthesize ECGs. Each Gaussian pulse is characterized by three parameters, namely amplitude, location and width. The method described in [9] is also based on Gaussian functions and estimates their parameters via nonlinear least square methods.

\section{VPW-FRI}

VPW-FRI is an extension of classical FRI where streams of Diracs are generalized by adding two parameters: width and asymmetry. Equivalently, we allow the roots of the annihilating filter to be located inside the unit circle (so that the filter is stable). To this end, let us introduce a new parameter $a_{k} \geq 0$ that we call width and write the roots as follows:

$$
u_{k}=e^{-2 \pi\left(a_{k}+i t_{k}\right) / \tau} \text {. }
$$

We define:

$$
X^{(1)}[m]=\frac{1}{\tau} \sum_{k=0}^{K-1} c_{k} e^{-2 \pi\left(a_{k}|m|+i t_{k} m\right) / \tau}, \quad m \in \mathbb{Z}
$$

and $X^{(2)}[m]$, which is the Hilbert transform of $X^{(1)}[m]$ (the amplitude of which is denoted by $d_{k}$ ):

$$
X^{(2)}[m]=-\frac{1}{\tau} \sum_{k=0}^{K-1} d_{k} \operatorname{sgn}(m) e^{-2 \pi\left(a_{k}|m|+i t_{k} m\right) / \tau}, m \in \mathbb{Z} .
$$

Let $X[m]=X^{(1)}[m]+X^{(2)}[m]$. If we consider the signal $X[m]$ for $m$ positive, we see that it is annihilated by the filter $A(z)=\prod_{k=0}^{K-1}\left(1-u_{k} z^{-1}\right)$. In this case, the annihilating filter is computed using (2) for $m>K$ and we can extract the parameters $\left\{t_{k}\right\}_{k=0}^{K-1}$ from the roots of $A(z)$ as in the case of streams of Diracs. Moreover, the widths $\left\{a_{k}\right\}_{k=0}^{K-1}$ are given by $a_{k}=\frac{\tau \log \left|u_{k}\right|}{2 \pi}$. Finally, the parameters $\left\{c_{k}\right\}_{k=0}^{K-1}$ and $\left\{d_{k}\right\}_{k=0}^{K-1}$ can be retrieved by solving equation (3) over the complex numbers and letting $c_{k}=\Re\left\{b_{k}\right\}$ and $d_{k}=-\Im\left\{b_{k}\right\}$.

The continuous-time $\tau$-periodic signal $x(t)$ is obtained by taking the inverse Fourier transform of $X[m]^{1}$ :

$$
\begin{aligned}
x(t)= & \sum_{k=0}^{K-1} x_{k}(t)=\sum_{k=0}^{K-1}\left(x_{k}^{(1)}(t)+x_{k}^{(2)}(t)\right) \\
= & \sum_{k=0}^{K-1} \sum_{n \in \mathbb{Z}} c_{k} \frac{a_{k}}{\left.\pi\left(a_{k}^{2}+\left(t-t_{k}-n \tau\right)^{2}\right)\right)} \\
& +\sum_{k=0}^{K-1} \sum_{n \in \mathbb{Z}} d_{k} \frac{t-t_{k}-n \tau}{\left.\pi\left(a_{k}^{2}+\left(t-t_{k}-n \tau\right)^{2}\right)\right)},
\end{aligned}
$$

\footnotetext{
herein.
} 


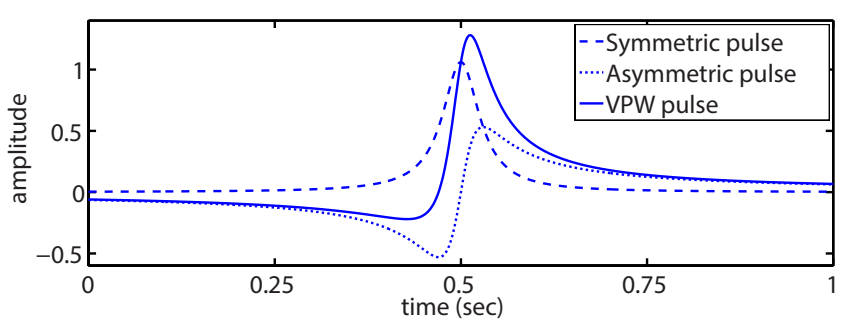

Fig. 1: Dashed line: symmetric pulse (Cauchy-Lorentz function), dotted line: asymmetric pulse, continuous line: VPW pulse. The parameters used are $t_{0}=0.5, a_{0}=0.03$ and $c_{0}=d_{0}=0.1$; only one period is shown $(n=0)$.

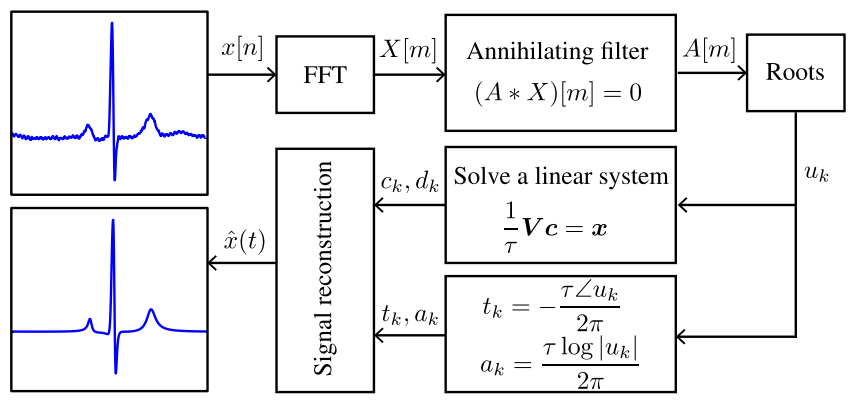

Fig. 2: VPW-FRI parameter estimation.

where $x_{k}^{(1)}(t)$ is the periodic expansion of symmetric pulses and $x_{k}^{(2)}(t)$ the periodic expansion of asymetric pulses (cf. Figure 1). An alternative formula for $x_{k}(t)$ that avoids the infinite sum is given by ${ }^{1}$ :

$$
x_{k}(t)=\frac{c_{k}}{\tau} \frac{1-\left|z_{t}\right|^{2}}{\left(1-z_{t}\right)\left(1-z_{t}^{*}\right)}+\frac{d_{k}}{\tau} \frac{2 \Im\left\{z_{t}\right\}}{\left(1-z_{t}\right)\left(1-z_{t}^{*}\right)}
$$

where $z_{t}=e^{2 \pi\left(-a_{k}+i\left(t-t_{k}\right)\right) / \tau}$. Like the Dirac pulse in traditional FRI, $x_{k}(t)$ is the atom for this class of signals and we call it VPW pulse. The block diagram in Figure 2 illustrates the VPW-FRI parameter estimation procedure.

Remark: VPW pulses are indeed a generalization of Diracs. By setting $d_{k}=0$ and taking the limit of $x_{k}(t)$ when $a_{k}$ goes to zero, we retrieve a Dirac with amplitude $c_{k}$ located at time $t_{k}$. Consequently, we can represent a wider range of signals by weighted sums of VPW pulses.

\section{ECG SIGNALS}

We apply the VPW-FRI modeling framework to electrocardiogram (ECG) signals. Each heart beat is decomposed into a sum of five pulses, called $P, Q, R, S, T$ pulses in the medical literature [11]. The $P$ wave characterizes the beginning of the heart beat and is generated by the depolarization of the atria. It is followed by the $Q R S$ complex, which corresponds to the depolarization of the ventricles; this is represented by three narrow and sharp pulses. The last pulse is the $T$ wave, which corresponds to the re-polarization of the cells.
Our modeling/compression uses VPW pulses to efficiently parametrize ECGs: Figure 3 shows a sum of five VPW pulses approximating a single heart beat of an ECG.

\subsection{Practical considerations}

We discuss various considerations regarding a practical implementation of VPW-FRI for ECG compression and we propose fixes to improve stability and boost the performance of the system.

First, it is important to pre-process the data to account for measurement noise and model mismatch. There are two denoising methods commonly used in the FRI framework: Cadzow's method [12] and the matrix-pencil algorithm [13], which is based on ESPRIT [14]. Both techniques also apply to VPW-FRI and empirical results show that the matrix-pencil algorithm leads to better performance.

Second, when processing multi-beat records, we need to segment the input signal before performing parameter estimation. A simple approach is to process each heart beat separately; in order to extract a single beat, QRS detection is needed [11]. Once the QRS locations have been identified, every beat is extracted so that the starting and ending points are located exactly between two consecutive QRS locations. To allow for a smoother transition between two consecutive reconstructed beats, it is necessary that the extracted heart beats slightly overlap. Thus, when stitching back the segments together, we can smooth any discontinuities by proper windowing.

Third, we need to ensure that the roots of the annihilating filter are indeed located inside the unit circle so that the model is stable. This is not guaranteed to be the case in practice due to noisy measurements and model mismatch. Let $\hat{a}_{k}$ be the estimated value of the parameter $a_{k}$. One way to cope with instability is to let $\hat{a}_{k} \leftarrow\left|\hat{a}_{k}\right|$. Another way to deal with this issue is to bring back the unstable roots at a fixed distance inside the unit circle; in other words, we set $\hat{a}_{k}=R$ if $\hat{a}_{k}<R$, for fixed threshold $R$. Finally, we can simply discard the roots located outside the unit circle. We noticed that fixing the amplitude performs the best, cf. Table 1 .

Last, recall that one of the main underlying assumptions is that the input signals are periodic in time. This is not exact

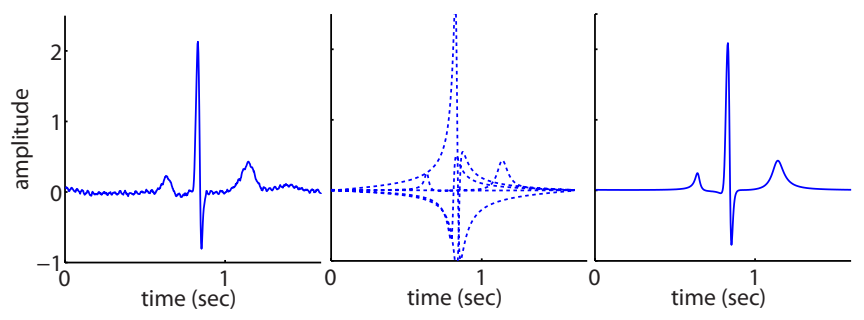

Fig. 3: Left: one period of a pulse (sample 123 of MIT-BIH arrhythmia database), middle: decomposition into $5 \mathrm{VPW}$ pulses, right: sum of the 5 pulses. 


\begin{tabular}{lr}
\hline Method & \multicolumn{1}{c}{ SRR } \\
\hline Plain (no correction) & $<-10 \mathrm{~dB}$ \\
Magnitude correction when $a_{k}<0$ & $11.77 \mathrm{~dB}$ \\
Magnitude correction when $a_{k}<1 / 200$ & $16.44 \mathrm{~dB}$ \\
Discard all roots s.t. $a_{k}<0$ & $11.77 \mathrm{~dB}$ \\
Discard all roots s.t. $a_{k}<1 / 200$ & $11.99 \mathrm{~dB}$ \\
\hline
\end{tabular}

Table 1: Different methods to obtain a stable annihilating filter; reported SRR is the average SRR obtained on 48 records (first 30 seconds) from the MIT-BIH arrhythmia database.

in real data for various reasons: for instance, breathing causes baseline wandering in the input signal. To improve the accuracy and reliability of the algorithm, we modify the extracted beat so that the amplitudes of the starting and ending points are the same. The correction is performed by subtracting a monotone signal from the extracted beat, e.g., one half of the period of a cosine. Our experiments using various recordings from the MIT-BIH arrhythmia database [15] show a noticeable improvement when performing such correction.

\section{SIMULATIONS}

For measuring the modeling accuracy of VPW-FRI, we define the signal to residual ratio (SRR) as

$$
\mathrm{SRR}:=20 \log _{10}\left(\frac{\|x\|_{2}}{\|x-\widehat{x}\|_{2}}\right),
$$

where $x$ is the original signal and $\widehat{x}$ denotes the reconstructed version. Furthermore, we define the compression ratio (CR) as the number of bits used to store signal $x$ divided by the number of bits used to store $\widehat{x}$. Ignoring errors due to quantization, a simplified way of measuring the compression is to record the number of parameters needed per second.

\subsection{Comparison with other techniques}

An example of a compressed ECG signal using VPW-FRI is shown in Figure 4. Overall, we observe that VPW-FRI is capable of preserving the morphological information of the signal, while discarding the high frequency noise present in most of ECG signals.

First, we compare the VPW-FRI model against Gaussianbased models. The two main differences between Gaussian and VPW pulses are that VPW pulses have heavier tails and, more importantly, they naturally incorporate asymmetry thus leading to a wider range of possible shapes at the cost of an extra parameter per pulse. The method described in [9] has been tested on the MIT-BIH database and a total of sixteen Gaussian pulses (48 parameters) is used to model a single heart beat. On randomly chosen sample beats, a root mean squared (RMS) error between 0.020 and 0.059 was claimed.

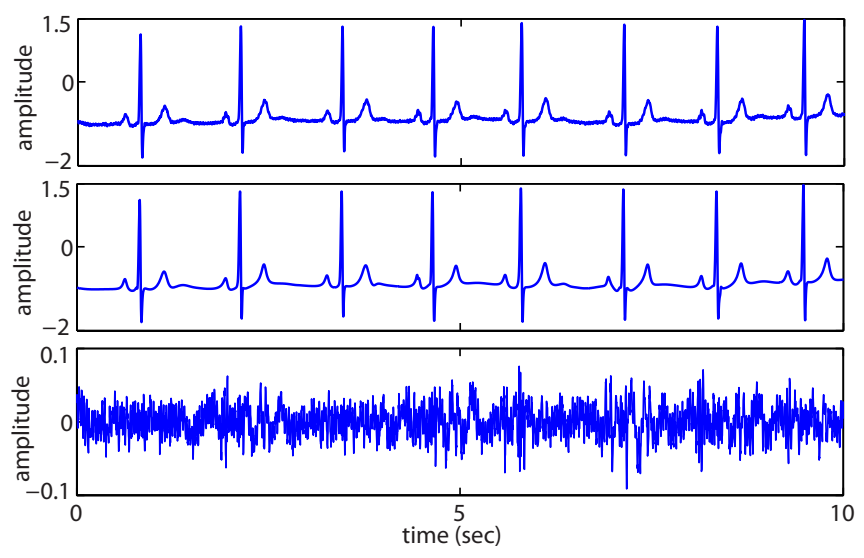

Fig. 4: Top: Record 123 from the MIT-BIH arrhythmia database, middle: signal reconstructed using VPW and $K=$ 7 (CR of 15), bottom: reconstruction error.

We obtain comparable results with seven VPW pulses (28 parameters per heart beat): indeed we observe an RMS error between 0.014 and 0.068 and an average RMS error of 0.027 (average SRR of $22.0 \mathrm{~dB}$ ) on the first 100 seconds of all 48 records of the MIT BIH database. The resulting CR is 9.5 in our case, compared to 5.5 in [9].

Second, we compare the compression performance of VPW against AZTEC [4]. Both methods are tested on the first 60 seconds of the entire MIT-BIH arrhythmia database. For a fixed CR, the resulting average SRR of VPW-FRI compression is $15 \mathrm{~dB}$ better than the average SRR of AZTEC.

Last, we compare our results to [6], where a CS algorithm is applied to a selection of signals from the MIT-BIH arrhythmia database. Typically, for a CR of 10, an SRR slightly below $22 \mathrm{~dB}$ is claimed on the first 10 minutes of record 107 as opposed to $25.4 \mathrm{~dB}$ when using our model. To achieve the same SRR as VPW-FRI, the CS approach results in a CR of 8 . The results presented in this article are reproducible: http://rr.epfl.ch/42.

\section{CONCLUSIONS}

We have proposed a parametric model for ECG signals, namely VPW-FRI, where the morphology of the signal is specifically leveraged; using common practice, each heart beat is captured by a sum of 5 pulses (P,Q,R,S,T), each of which is modeled by a VPW pulse. We have analyzed a method for estimating the parameters and have provided various heuristics to boost the performance in the presence of noise and model mismatch. Extensive experiments have indicated improvements over current methods in terms of all modeling accuracy, compression efficiency and sampling rate. 


\section{REFERENCES}

[1] M. Vetterli, P. Marziliano, and T. Blu, "Sampling signals with finite rate of innovation," IEEE Transactions on Signal Processing, vol. 50, no. 6, pp. 1417-1428, jun. 2002.

[2] R. F. Quick, R. E. Crochiere, J. H. Hong, A. Hormati, and G. Baechler, "Extension of FRI for modeling of electrocardiogram signals," International Conference of the IEEE Engineering in Medicine and Biology Society, pp. 2909-2912, aug. 2012.

[3] S.M.S. Jalaleddine, C.G. Hutchens, R.D. Strattan, and W.A. Coberly, "ECG data compression techniques-a unified approach," IEEE Transactions on Biomedical Engineering, vol. 37, no. 4, pp. 329-343, apr. 1990.

[4] J.R. Cox, F.M. Nolle, H.A. Fozzard, and G.C. Oliver, "AZTEC, a preprocessing program for real-time ECG rhythm analysis," IEEE Transactions on Biomedical Engineering, vol. BME-15, no. 2, pp. 128-129, apr. 1968.

[5] M. Nakashizuka, H. Kikuchi, H. Makino, and I. Ishii, "ECG data compression by multiscale peak analysis," in IEEE International Conference on Acoustics, Speech, and Signal Processing, may 1995, vol. 2, pp. 11051108.

[6] Luisa F. Polania, Rafael E. Carrillo, Manuel BlancoVelasco, and Kenneth E. Barner, "Compressed sensing based method for ECG compression," in IEEE International Conference on Acoustics, Speech, and Signal Processing, may 2011, pp. 761-764.

[7] Y. Hao, P. Marziliano, M. Vetterli, and T. Blu, "Compression of ECG as a signal with finite rate of innovation," in 27th Annual International Conference of the Engineering in Medicine and Biology Society, jan. 2005, pp. 7564-7567.

[8] P.E. McSharry, G.D. Clifford, L. Tarassenko, and L.A. Smith, "A dynamical model for generating synthetic electrocardiogram signals," IEEE Transactions on Biomedical Engineering, vol. 50, no. 3, pp. 289-294, mar. 2003.

[9] M.S. Billah, T.B. Mahmud, F.S. Snigdha, and M.A. Arafat, "A novel method to model ECG beats using gaussian functions," in International Conference on Biomedical Engineering and Informatics (BMEI), oct. 2011, vol. 2, pp. 612-616.

[10] G. Baechler, "Sensing ECG signals with variable pulse width finite rate of innovation," M.S. thesis, EPFL, 2012.
[11] L. Sörnmo and P. Laguna, Bioelectrical Signal Processing in Cardiac and Neurological Applications, Elsevier Academic Press, jun. 2005.

[12] J.A. Cadzow, "Signal enhancement-a composite property mapping algorithm," IEEE Transactions on Acoustics, Speech and Signal Processing, vol. 36, no. 1, pp. 49-62, jan. 1988.

[13] Y. Hua and T.K. Sarkar, "Matrix pencil method for estimating parameters of exponentially damped/undamped sinusoids in noise," IEEE Transactions on acoustics, speech and signal processing, vol. 38, no. 5, pp. 814824, may 1990.

[14] R. Roy and T. Kailath, "ESPRIT-estimation of signal parameters via rotational invariance techniques," IEEE Transactions on Acoustics, Speech and Signal Processing, vol. 37, no. 7, pp. 984-995, jul. 1989.

[15] A.L. Goldberger et al., "Physiobank, physiotoolkit, and physionet : Components of a new research resource for complex physiologic signals," Circulation, vol. 101, no. 23, pp. 215-220, 2000. 